\title{
13. Gesetzgebung über den Kunsthandel in der Schweiz
}

\section{Catherine Schümperli Younossian}

\section{(2) OpenEdition \\ 1 Journals}

Electronic version

URL: http://journals.openedition.org/sjep/232

DOI: 10.4000/sjep.232

ISSN: 1663-9677

Publisher

Institut de hautes études internationales et du développement

\section{Printed version}

Date of publication: 1 avril 2004

Number of pages: 233-242

ISSN: 1660-5926

\section{Electronic reference}

Catherine Schümperli Younossian, «13. Gesetzgebung über den Kunsthandel in der Schweiz »,

Schweizerisches Jahrbuch für Entwicklungspolitik [Online], 23-1 | 2004, Online erschienen am: 29 April

2010, abgerufen am 08 September 2020. URL : http://journals.openedition.org/sjep/232 ; DOI :

https://doi.org/10.4000/sjep.232 


\section{Gesetzgebung über den Kunsthandel in der Schweiz*}

D

IE SCHWEIZ spielt im internationalen Kunsthandel eine wichtige Rolle: Sie belegt unter den auf dem Kunstmarkt aktiven Staaten den vierten Rang. Allerdings steht die Schweiz auch im Ruf, eine „Drehscheibe“ für den illegalen Kulturgüterhandel zu sein. Genährt wird diese Reputation vor allem dadurch, dass die Schweiz keine einschlägigen Gesetze auf Bundesebene besass und dass sie die wichtigsten internationalen Übereinkommen über den Kulturgüterhandel nicht ratifiziert hatte. Es dauerte fast fünfzehn Jahre, um die entgegengesetzten Positionen der wichtigsten Akteure (Kunsthändler und -sammler einerseits, Kulturkreise und Entwicklungsorganisationen andererseits) auf einen gemeinsamen Nenner zu bringen und dem schweizerischen Parlament einen Gesetzesentwurf zu unterbreiten. So verabschiedeten die Eidgenössischen Räte im Juni 2003 das Bundesgesetz über den internationalen Kulturgütertransfer (KGTG) und stimmten der Ratifizierung der internationalen UNESCO-Konvention von 1970 zu. Vor allem die Kulturkreise und die Entwicklungsorganisationen hatten dafür plädiert, die Spielregeln für den Kulturgüterverkehr in der Schweiz neu zu definieren und insbesondere das Kulturerbe der Entwicklungsländer zu bewahren, namentlich wenn Kulturgüter im Bereich Archäologie und Ethnologie betroffen sind.

Seine Besonderheit verdankt der Kunsthandel nicht nur den gehandelten Gegenständen, sondern auch der Tatsache, dass er zu einem bedeutenden Teil von illegalen Machenschaften beherrscht wird. Der Bundesrat äussert sich in seiner Botschaft zur Besonderheit der Kulturgüter wie folgt ${ }^{1}$ : „Sie sind Identifikationsträger für die Einzelnen und die Gemeinschaft, fassbare Zeugnisse der Kultur und Geschichte, in ihrer Bedeutung einzigartig und unersetzlich. Sie prägen das Selbstverständnis und den sozialen Zusammenhalt einer Gesellschaft" ${ }^{* 2}$. Weiter steht in der Botschaft: „Die grosse Nachfrage nach archäologischen und ethnologischen Objekten hat dazu geführt, dass sich auch der illegale Handel zu einem sehr lukrativen Geschäft entwickelte und vielerorts von der organisierten Kriminalität übernommen wurde“. „Er geht einher mit Diebstahl, mit der Plünderung und Zerstörung archäologischer Stätten [...]. Darunter zu leiden haben alle Länder, insbesondere aber kulturgüterreiche Regionen, in denen die Mittel oder staatlichen Strukturen für einen effizienten Schutz nicht ausreichen“3.

* Von Catherine Schümperli Younossian, Forschungsbeauftragte am iuéd.

1 Bundesrat, Botschaft über die UNESCO-Konvention 1970 und das Bundesgesetz über den internationalen Kulturgütertransfer (KGTG) vom 21. November 2001 (BB1 2002 535).

2 Ibid., S. 538.

Ibid. 


\section{$\square$ Einige Zahlen}

In den letzten Jahrzehnten hat der internationale Kulturgüterhandel global an Bedeutung gewonnen. Eine 2004 veröffentlichte Studie ${ }^{4}$ enthält aufschlussreiche Angaben über den internationalen Kunsthandel insgesamt und über den relativen Anteil der verschiedenen Länder. Der Autor der Studie weist darauf hin, dass der Weltmarkt zwischen 1970 und Mitte der 80er Jahre global ein kräftiges Wachstum aufweist: Die Ein- und Ausfuhren stiegen von knapp 500 Millionen Dollar auf 3 bis 4 Milliarden an. Während der zweiten Hälfte der 80er Jahre erlebte der Markt einen Boom und erreichte 1990 mit Importen und Exporten bei 14 Milliarden Dollar Höchstwerte. Ab 1991 setzte ein drastischer Einbruch ein, und der weltweite Handel schrumpfte auf die Hälfte des Standes von 1990. Seither wird auf dem Markt eine zögerliche Erholung verzeichnet. Die Schwankungen auf dem Kunstmarkt gehen hauptsächlich auf das beispiellose Wachstum der Auktionshäuser in den Jahren 1985 bis 1989 zurück, welches mit der Explosion der Nachfrage auf dem internationalen Markt und mit dem ab 1987 exponentiellen Preisanstieg zusammenfiel. Der Börsenkrach vom Oktober 1987, der Mini-Krach vom Oktober 1989, der Golfkrieg 1990, die Wirtschaftsflaute und - direkt den Kunsthandel betreffend - der Rückzug der japanischen Käuferschaft dämpften die Nachfrage nach Kunstwerken. In der Saison 1990-1991 verzeichnete Sotheby's im Vergleich zum Vorjahr Umsatzeinbussen von 59 Prozent, Christie's von 49 Prozent und das Hôtel Druot von 43 Prozent ${ }^{5}$.

In puncto Stellenwert der einzelnen Länder geht aus der Studie von Sébastien Guex $^{6}$ hervor, dass die Vereinigten Staaten mit einem Marktanteil von rund einem Drittel den weltweiten Kunstmarkt dominieren und das Vereinigte Königreich, das ungefähr ein Viertel ausmacht, deutlich überrunden. Wie zudem aus der Studie zu ersehen ist, folgt mit grossem Abstand ein wahrscheinlich von der Schweiz angeführtes Trio. Der wegen der Bedeutung des Graumarktes und/oder illegalen Handels sicherlich unterschätzte Anteil der Schweiz liegt bei 9 Prozent, dicht gefolgt von Deutschland (rund $8 \%$ ) und Frankreich $(5 \%)^{7}$. In diesen Zahlen wird der illegale Transfer, dessen Jahreseinnahmen schätzungsweise 6 Milliarden Dollar betragen, selbstverständlich nicht berücksichtigt ${ }^{8}$.

\section{$\square$ Die Schweiz und der Kunsthandel}

Gemäss der Aussenhandelsstatistik der Schweiz lagen die Einfuhren von Kulturgütern im Jahr 2002 bei über 1,1 Milliarden Franken und die Ausfuhren im gleichen Jahr bei 1,3 Milliarden Franken.

4 S. Guex, „Le marché suisse de l'art 1886-2000. Un survol chiffré“, in Guex S. und Lafontant Vallotton C. (dir), Le marché suisse de l'art (XIX et XX siècles), Traverse. Revue d'histoire, Nr. 1, 2002, S. 29-62.

5 R. Moulin, Le marché de l'art, coll. Champs, Paris, Flammarion, 2003.

Le marché suisse de l'art..., op. cit.

Ibid., S. 34.

„Internet et Interpol volent au secours du patrimoine culturel africain“, Le Temps, 31. Juli 2000. 
Tabelle 38: Schweizerische Importe und Exporte von Kunstwerken und Antiquitäten, 1999-2002 (in Millionen Schweizer Franken)

\begin{tabular}{lrrrr}
\hline & $\mathbf{1 9 9 9}$ & $\mathbf{2 0 0 0}$ & $\mathbf{2 0 0 1}$ & $\mathbf{2 0 0 2}$ \\
\hline Importe & 1002 & 1359 & 1594 & 1106 \\
\hline Exporte & 955 & 1435 & 1101 & 1337 \\
\hline
\end{tabular}

Quelle: Oberzolldirektion, Schweizerische Aussenhandelsstatistik. Jahresbericht 2002, Bern, 2003.

$\mathrm{Zu}$ den Gründen, welche die Schweiz $\mathrm{zu}$ einem führenden internationalen Kunsthandelsplatz gemacht haben, gehen die Meinungen auseinander. In der Botschaft des Bundesrates steht dazu: „Dies verdankt sie in erster Linie der Erfahrung und Kompetenz ihrer Kunsthändlerinnen und Kunsthändler. Sie wissen die attraktiven Rahmenbedingungen zu nutzen, die sich aus ihrer zentralen Lage, der ausgebauten Infrastruktur und den gut etablierten internationalen Kontakten ergeben“. Allerdings hält die Botschaft auch Folgendes fest: „Die Schweiz steht im Ruf, eine Drehscheibe des illegalen Kulturgütertransfers zu sein" "9. Tatsächlich haben in den letzten Jahren zahlreiche Diebstähle und Fälle von Hehlerei in der nationalen und internationalen Presse Schlagzeilen gemacht und die Schweiz ins Kreuzfeuer der Kritik gestellt. Ein weiteres Element, das diese Rolle der Schweiz tendenziell belegt, ist die wachsende Anzahl von Rechtshilfegesuchen zu gestohlenen oder illegal ausgeführten Kulturgütern ${ }^{10}$.

Bis zur jüngsten Verabschiedung des im vorliegenden Artikel beschriebenen Bundesgesetzes über den internationalen Kulturgütertransfer (KGTG) verfügte die Schweiz über einen lockeren Rechtsrahmen für den Kunsthandel. Im Gegensatz zu anderen wichtigen Kunsthandelsnationen besass die Schweiz auf Bundesebene keine spezifischen, auf den Kulturgüterverkehr anwendbaren Regeln; dieser Bereich fällt laut Bundesverfassung in die Zuständigkeit der Kantone. Ebensowenig war die Schweiz den internationalen Instrumenten unterstellt, welche den Kulturgütertransfer regeln (siehe unten). In den Zollfreilagern (Genf, Zürich und Basel) konnten Kulturgüter auf wenig transparente Weise importiert, exportiert und zwischengelagert werden (so fehlte z.B. die Pflicht, ein Register der gelagerten Güter zu führen). Dieses Rechtsdefizit leistete dem schlechten Ruf der Schweiz Vorschub ${ }^{11}$.

\section{$\square$ Internationale Übereinkommen über den Kulturgüterhandel}

Auf internationaler Ebene existieren zwei Konventionen über den Kulturgüterhandel, nämlich die Konvention der UNESCO vom 14. November 1970 über die Massnahmen zum Verbot und zur Verhütung der unzulässigen Einfuhr, Ausfuhr und Übereignung von Kulturgut ${ }^{12}$ und die Unidroit-Konvention ${ }^{13}$ vom 24. Juni 1995 über gestohlene oder rechtswidrig ausgeführte Kulturgüter.

\section{$\square$ Die UNESCO-Konvention von 1970}

Die UNESCO-Konvention fordert die Rückgabe von gestohlenen und rechtswidrig ausgeführten Kulturgütern. Die Konvention ist nicht rückwirkend; sie betrifft

\footnotetext{
Botschaft über die UNESCO-Konvention von 1970..., op. cit., S. 545.

Ibid.

„Visite guidée dans les ports francs de Genève“, Le Temps, 24. November 2003.

Informationen zur Konvention : <www.unesco.org/culture/laws/1970/html_fr/page1.shtml>.

3 Internationales Institut zur Vereinheitlichung des Privatrechts (Unidroit) : <www.unidroit.org $>$.
} 
nur Objekte aus den Beständen von Museen und vergleichbaren Einrichtungen. Die Signatarstaaten verpflichten sich, die Aus- und Einfuhr von Kulturgütern zu kontrollieren, an der Erstellung von Inventaren mitzuwirken und sich über die Behandlung der Objekte auf dem Laufenden zu halten. Des Weiteren verpflichten sie sich zu gegenseitiger Unterstützung bei der Aufdeckung illegaler Transaktionen. Ende 2003 waren 102 Staaten der Konvention beigetreten, darunter zahlreiche Länder des Südens sowie neun Mitglieder der Europäischen Union (Dänemark, Spanien, Finnland, Frankreich, Griechenland, Italien, Portugal, Vereinigtes Königreich, Schweden), ferner Australien, die Vereinigten Staaten und Kanada. Die Bestimmungen der Konvention sind nicht direkt anwendbar (non self-executing) und müssen deshalb zuerst formell in das nationale Recht der einzelnen Vertragsstaaten umgesetzt werden. Die Konvention wurde in verschiedener Hinsicht kritisiert. Hinsichtlich der Definition der Kulturgüter lässt die sehr allgemeine Formulierung einen grossen Handlungsspielraum für die Umsetzung in das nationale Recht offen. Bestimmte Kreise werfen dem Text eine zu einseitige Ausrichtung vor; das erklärt den geringen Anklang bei „Import“-Staaten von Kulturgütern. Ausserdem besitzt die Konvention einen stark eingeschränkten Geltungsbereich, da sie sich nur mit der Rückgabe von in Museen oder ähnlichen Einrichtungen gestohlenen Kulturgütern befasst. Im Bewusstsein um diese Einschränkungen hatte die UNESCO das Internationale Institut zur Vereinheitlichung des Privatrechts (Unidroit) aufgefordert, sich mit dem privatrechtlichen Bereich auseinander zu setzen. So entstand die Unidroit-Konvention vom 24. Juni 1995 über gestohlene oder rechtswidrig ausgeführte Kulturgüter.

\section{Die Unidroit-Konvention}

Der Kunsthandel spielt sich im Wesentlichen im privaten Bereich ab (Verkaufsverträge, Übereignung an gutgläubige Erwerber usw.). Aus diesem Grund wurde es unumgänglich, die Frage im Privatrecht zu behandeln. Ziel ist es, zu vermeiden, dass die interessierten Kreise die Vielfalt der in den verschiedenen Ländern geltenden Gesetze ausnutzen; gleichzeitig sollen die Grundsatzerklärungen, auf der die UNESCO-Konvention beruht, wirksam weiterentwickelt werden. Die Unidroit-Konvention ermöglicht es, illegale Machenschaften im internationalen Kulturgüterhandel zu bekämpfen. Parallel dazu stellt sie allen rechtmässigen Eigentümern - Staaten, Museen oder Sammlern - ein juristisches Instrumentarium für die Durchsetzung ihrer Rechte zur Wiedererlangung von gestohlenen oder rechtswidrig ausgeführten Kulturgütern vor ordentlichen Gerichten zur Verfügung. Wenn demnach ein ausländischer Besitzer oder Staat Rückgaberechte vorbringen würde, müssten die schweizerischen Gerichte und Richter über die Legitimität der Ansprüche befinden.

Im Gegensatz zur UNESCO-Konvention ist die Unidroit-Konvention direkt anwendbar (self-executing), d.h. ihre Bestimmungen müssen nicht in das nationale Recht der Vertragsparteien umgesetzt werden. So werden in einem allgemein verbindlichen Dokument die Bedingungen für die Garantie der Rückgabe bzw. Rückführung von gestohlenen oder rechtswidrig ausgeführten Kulturgütern sowie das Verfahren für die Durchsetzung der Rechtsansprüche auf internationaler Ebene niedergelegt. Zwei Punkte verdienen besondere Erwähnung: Die Konvention ist erstens nicht rückwirkend und gilt somit nicht für Kulturgüter, die vor ihrem In-Kraft-Treten gestohlen oder illegal ausgeführt wurden. Zweitens sieht sie eine angemessene Entschädigung für den gutgläubigen Besitzer 
eines gestohlenen oder rechtswidrig ausgeführten Kulturgutes vor, wenn dieser das fragliche Objekt zurückgegeben hat.

Im Juni 1996 setzte der Bundesrat eine Arbeitsgruppe ein, die bestimmte Rechtsfragen klären sollte, welche in den zwei Konventionen aufgeworfen werden. Die interdepartementale Arbeitsgruppe verfasste einen Bericht mit dem Titel Internationaler Kulturgütertransfer ${ }^{14}$. In den Schlussfolgerungen des Berichts wird festgehalten, dass ,die Probleme im internationalen Kulturgüterverkehr mit Unidroit wirksam angegangen werden können. Eine Ratifikation von UNESCO rechtfertigt sich insbesondere deshalb, weil damit ein multilateraler Rahmen für eine zwischenstaatliche Zusammenarbeit auf dem Gebiet des internationalen Kulturgüterschutzes geschaffen wird“". Ausserdem wird betont, dass weder verfassungs- noch privatrechtliche Hindernisse der Ratifizierung der beiden Konventionen entgegenstünden. Die Arbeitsgruppe sprach sich für die Ratifizierung der beiden Konventionen und für die Ausarbeitung von Gesetzen zu deren Umsetzung auf innerstaatlicher Ebene aus.

Der Bundesrat schloss sich indessen den Schlussfolgerungen der Expertengruppe nicht an. Die Botschaft, die er den Eidgenössischen Räten unterbreitete, betrifft ausschliesslich die Ratifizierung der UNESCO-Konvention von 1970 und das Umsetzungsgesetz auf Bundesebene. Für die Unidroit-Konvention hält der Bundesrat eine Entscheidung zur Ratifikation für verfrüht. Das EDI wurde aber beauftragt, „die internationale Entwicklung zu beobachten und zu gegebener Zeit dem Bundesrat entsprechende Vorschläge zu unterbreiten" ${ }^{15}$.

\section{$\square$ Langwieriger Gesetzgebungsprozess}

Seit der Bildung der ersten Arbeitsgruppe 1990, die sich mit der Frage der Exporte der schweizerischen Kulturgüter und der Importe ausländischer Kulturgüter beschäftigte, dauerte es rund dreizehn Jahre, bis die Eidgenössischen Räte im Juni 2003 ein Gesetz zur Regelung der Materie verabschiedeten. Offensichtlich war diese Zeit nötig, um eine Annäherung zwischen den beiden sich gegenüberstehenden Lagern herbeizuführen: einerseits den Befürwortern der doppelten Ratifizierung der beiden internationalen Konventionen (UNESCO und Unidroit) und einer strengen schweizerischen Gesetzgebung (im Wesentlichen Kulturkreise und Entwicklungs-NGO) und andererseits bestimmten Händlerund Sammlerkreisen, nach deren Auffassung ein Gesetz grosse Einschränkungen schaffen und ihre Tätigkeit behindern würde.

Zahlreiche Vernehmlassungsverfahren und öffentliche Anhörungen waren erforderlich $^{16}$, bis das Bundesamt für Kultur schliesslich einen Gesetzesentwurf unterbreiten konnte, der von den betroffenen Kreisen nicht grundsätzlich abgelehnt wurde.

14 Bundesamt für Kultur, Internationaler Kulturgüterverkehr, Bericht der Arbeitsgruppe, Nr. 304.250 d, BAK, Bern, September 1998. Vertrieb: Eidgenössische Drucksachen- und Materialzentrale, 3000 Bern. Im Internet: <www.kultur-schweiz.admin.ch/arkgt/kgt/index_.htm>, unter ,Unsere Publikationen“.

15 Eidgenössisches Departement des Innern, Bericht der Arbeitsgruppe Internationaler Kulturgütertransfer liegt vor, Pressemitteilung, 26. August 1998.

16 Vernehmlassungsverfahren betreffend die UNESCO-Konvention von 1970 (1993), Vernehmlassungsverfahren betreffend die Unidroit-Konvention (1996), Interdepartementale Arbeitsgruppe (19961998), Öffentliche Anhörungen (1999). Für weitere Einzelheiten wird auf die Botschaft über die UNESCO-Konvention von $1970 \ldots$, op. cit. verwiesen. 
Der vehemente Widerstand der Kunstkreise gegen den Gesetzesentwurf schlug sich in der als Gegenentwurf eingereichten Parlamentarischen Initiative Fischer nieder ${ }^{17}$. Diese beantragte eine Schwächung des KGTG (besonders betreffend die Rückgabefrist, die nur zehn Jahre dauern soll, und die Lockerung der Meldepflicht zur Herkunft importierter Kulturgüter). Dagegen schlossen sich 21 schweizerische NGO in einer von der Erklärung von Bern ${ }^{18}$ koordinierten Plattform zusammen und appellierten an die Schweizer Parlamentarier, für den Entwurf zu stimmen, erinnerten aber auch daran, dass die Unterzeichnung der Unidroit-Konvention einen weiteren Meilenstein für den fairen Kulturgüterhandel darstelle ${ }^{19}$. Die Schweizerische UNESCO-Kommission ${ }^{20}$ bildete die Speerspitze der Bewegung der Kulturkreise für die Förderung der Ratifizierung der beiden Konventionen und für den Appell nach einem Umsetzungsgesetz.

\section{$\square$ Botschaft des Bundesrates}

Der Bundesrat beantragte den Eidgenössischen Räten in der (unten beschriebenen) Botschaft vom 21. November 2001, die UNESCO-Konvention von 1970 über die Massnahmen zum Verbot und zur Verhütung der unzulässigen Einfuhr, Ausfuhr und Übereignung von Kulturgut zu ratifizieren, und unterbreitete ihnen den Entwurf eines Bundesgesetzes über den internationalen Kulturgütertransfer (KGTG).

Mit dem Antrag an das Parlament, der Ratifizierung der UNESCO-Konvention und dem Umsetzungsgesetz zuzustimmen, betonte der Bundesrat die Bedeutung des Kunsthandels, äusserte aber einen klaren Wunsch: „Das Kulturgütertransfergesetz soll den illegalen und ethisch verwerflichen Geschäften mit Kulturgütern in der Schweiz einen Riegel schieben. Die Missbräuche werden gezielt bekämpft"'21.

\section{Bundesgesetz über den internationalen Kulturgütertransfer (KGTG)}

Das KGTG verfolgt zwei Hauptziele: zum einen den Schutz des kulturellen Erbes der Schweiz (mit der Aufforderung an den Bund und die Kantone, ein Verzeichnis der Kulturgüter zu erstellen, sowie mit dem Erlass von Bestimmungen über die Ausfuhr von Kulturgütern von wesentlicher Bedeutung), zum anderen die Einführung eines Rechtsrahmens für die Regelung des internationalen Kunsthandels.

Mit dem KGTG werden die Verpflichtungen aus der UNESCO-Konvention umgesetzt, für welche der Bund kompetent ist. Das Gesetz trägt namentlich folgenden Zielsetzungen Rechnung:

๖ Schaffung gesetzlicher Grundlagen, die es erlauben, Missstände auf dem Gebiet des Kulturgütertransfers zu bekämpfen;

$\square$ Verstärkung der internationalen Zusammenarbeit auf dem Gebiet des Kulturgütertransfers ;

17 Parlamentarische Initiative „Erlass eines Bundesgesetzes über den Transfer von Kulturgütern“, Nr. 01.450. Die Initiative wurde anlässlich der Frühjahrssession 2003 des Nationalrates eingereicht und nach den Beratungen im Nationalrat zurückgezogen.

18 Website der Erklärung von Bern : <www.EvB.ch>.

19 Stellungnahme der NGO zum Bundesgesetz über den internationalen Kulturgütertransfer (30. April 2002), einsehbar auf der Website der Erklärung von Bern : <www.EvB.ch>.

20 Website der Schweizerischen UNESCO-Kommission : <www.unesco.ch>.

${ }^{21}$ Botschaft über die UNESCO-Konvention von 1970..., op. cit., S. 537. 
- Anpassung der Regelungsmaterie an die international üblichen Mindeststandards ;

\ Förderung eines offenen und fairen internationalen Austausches von Kulturgut.

Das Gesetz enthält Regelungen zur Einfuhr, Ausfuhr und Übertragung von Kulturgut. Es sieht Massnahmen vor, die den Schutz des Kulturerbes der Schweiz und der übrigen Vertragsstaaten der UNESCO-Konvention verbessern sollen. Ausserdem wird der Kulturgüteraustausch gefördert. Einige wichtige Aspekte des Gesetzes werden in der Folge beschrieben :

$\square$ Zur Regelung der Einfuhr von Kulturgut in die Schweiz sieht das Gesetz zwei Massnahmen vor (Art. 7 und 8 KGTG): Erstens schliesst die Schweiz auf Antrag eines Vertragsstaates der UNESCO-Konvention mit diesem eine bilaterale Vereinbarung ab, welche die Einfuhr von Kulturgütern regelt, die für das Kulturerbe des fraglichen Staates von wesentlicher Bedeutung sind. Zweitens kann der Bundesrat zeitlich befristete Massnahmen zur Ein- und Ausfuhr von Kulturgut aus von ausserordentlichen Ereignissen (Kriege, Naturkatastrophen) betroffenen Gebieten beschliessen.

๖ Die Ausfuhr von Kulturgut wird ausschliesslich für den beschränkten Bereich der Kulturgüter im Besitz des Bundes geregelt (für die übrigen Kulturgüter liegt die Kompetenz bei den Kantonen). Es wird vorgesehen, ein Verzeichnis der Kulturgüter im Besitz des Bundes zu erstellen; dies bewirkt, dass sie dem Handel entzogen werden und ihre definitive Ausfuhr aus der Schweiz verboten ist. Der Zoll kontrolliert die Ausfuhr von Kulturgut aus der Schweiz (Art. 3 bis 6 und 19 KGTG).

๖ Zur Übertragung von Kulturgut formuliert das Gesetz Regeln, welche die Institutionen des Bundes beim Erwerb von Kulturgütern einzuhalten haben. Weiter legt das Gesetz Regeln für den Kunsthandel und das Auktionswesen fest. Die Händler sollen die Herkunft der Kulturgüter aufzeichnen, ihre Kundschaft über bestehende Ein- und Ausfuhrregelungen unterrichten und einer Fachstelle (die im Fall der Annahme des KGTG geschaffen werden muss) alle erforderlichen Auskünfte zur Erfüllung der Sorgfaltspflicht liefern (Art. 15 bis $17 \mathrm{KGTG).} \mathrm{Schliesslich} \mathrm{wird} \mathrm{die} \mathrm{Frist} \mathrm{für} \mathrm{die} \mathrm{Ersitzung} \mathrm{und}$ Rückgabe von gestohlenen Kulturgütern auf dreissig Jahre erhöht (Art. 32 KGTG). Besonders die Frage der Fristen hatte im Parlament lebhafte Diskussionen ausgelöst (siehe unten).

๖ Ergänzend sieht das KGTG die Möglichkeit vor, dass der Bund eine Finanzhilfe für die Erhaltung des kulturellen Erbes gewähren kann (Art. 14 KGTG). Das Gesetz enthält ausserdem flankierende Bestimmungen zu den Behörden (Art. 18 bis 20 KGTG), zur Amts- und Rechtshilfe (Art. 21 bis 23 KGTG) und zu den Strafen (Art. 24 bis 29 KGTG). Schliesslich ist vorgesehen, eine Fachstelle zu schaffen, die im Wesentlichen die Kantons- und Bundesbehörden beraten und unterstützen sowie zum Schutz des kulturellen Erbes mit den Behörden anderer Staaten zusammenarbeiten soll (Art. 18 KGTG).

\section{$\square$ Beratungen in den Eidgenössischen Räten}

Die Eidgenössischen Räte befassten sich anlässlich der Frühjahrs- und Sommersessionen 2003 mit dem Bundesbeschluss über die Genehmigung der UNESCOKonvention 1970 und mit dem Entwurf des KGTG. Abgesehen von der Defini- 
tion des Kulturgutes stimmt das Gesetz, das am 20. Juni 2003 verabschiedet wurde, mit dem Entwurf des Bundesrates weitgehend überein. Im Gesetzesentwurf hatte der Bundesrat das - schliesslich zu Grunde gelegte ${ }^{22}$ - Konzept Kulturgut breit definiert und zudem die Kulturgüter im engeren Sinn (archäologische Objekte, ethnologische Objekte, sakrale Gegenstände, Archivgut), für welche strenge Kontrollen verlangt wurden, genau umschrieben. Die Eidgenössischen Räte verzichteten auf die Doppeldefinition. Durch ihre Entscheidung wird der Geltungsbereich des Gesetzes de facto ausgedehnt.

Der Nationalrat befasste sich im März 2003 mit dem Gesetz, der Ständerat im Juni 2003. Drei Hauptpunkte wurden in den parlamentarischen Beratungen diskutiert $^{23}$ :

- Meldepflicht. Die Kommission des Nationalrates hatte beantragt, eine Meldepflicht für Fälle einzuführen, in denen ein begründeter Verdacht auf illegale Einfuhr von Kulturgütern oder ein Verdacht auf Handel mit Raubgut besteht. Der Antrag wurde von den linken Parteien und von den Christdemokraten unterstützt. Die Freisinnigen, die SVP und die Liberalen äusserten dagegen ihre Besorgnis zu einer Bestimmung, die dem Denunziantentum Vorschub leisten und die Kunsthändler mit der Situation konfrontieren würde, dass das Vertrauensverhältnis zwischen Händlern und Kunden untergraben wird. Nach der Abstimmung im Nationalrat wurde der Antrag aufgehoben.

- Verjährungsfrist. Der Bundesrat sah eine Verjährungsfrist von dreissig Jahren vor: Nach dieser Frist muss ein gutgläubig erworbenes Kulturgut dem Eigentümer nicht mehr obligatorisch zurückgegeben werden. Die dreissigjährige Frist entspricht der einschlägigen europäischen Regelung. Der Nationalrat folgte indessen dem Vorschlag eines SVP-Parlamentariers, wonach die Verjährungsfrist auf fünfzehn Jahre herabgesetzt werden sollte. Gegenüber dem Nationalrat verlangte der Ständerat die Erhöhung der Verjährungsfrist für die Rückgabe von Raubgut von den heute geltenden fünf auf dreissig Jahre.

- Entschädigungsart. Dieser Punkt betrifft die Art der Entschädigung eines gutgläubigen Erwerbers im Besitz von illegal eingeführtem Kulturgut. Der Nationalrat ging auf den Vorschlag einer liberalen Parlamentarierin ein, wonach bei der Rückgabe eines Kulturgutes an den Antrag stellenden Staat die Entschädigung nicht auf der Basis des Kaufpreises, sondern des Verkehrswerts festgesetzt wird. Der Ständerat hielt dagegen die Entschädigung auf der Basis des Verkehrswerts für mit der UNESCO-Konvention unvereinbar. Gemäss dem Ständerat würde diese Entschädigung die Spekulation anheizen und es einem finanzschwachen Staat erschweren, ihm zustehendes Kulturgut zurückzuerlangen. Deshalb zieht der Ständerat eine nach dem Kaufpreis berechnete Entschädigung vor.

Im Differenzbereinigungsverfahren schloss sich der Nationalrat sämtlichen Beschlüssen des Ständerates an. Das Gesetz wurde in der Schlussabstimmung am 20. Juni $2003^{24}$ angenommen und wird im Laufe des Jahres 2004 in Kraft treten.

22 Als „Kulturgut“ gilt ein aus religiösen oder weltlichen Gründen für Archäologie, Vorgeschichte, Geschichte, Literatur, Kunst oder Wissenschaft bedeutungsvolles Gut, das einer der Kategorien nach Artikel 1 der UNESCO-Konvention 1970 angehört (Art. 2 Abs. 1 KGTG).

23 Parlament, Zusammenfassung 01.077. Kulturgütertransfergesetz, 30. Juli 2003, <www.parlament.ch>.

24 Gesetzestext: Bundesgesetz über den internationalen Kulturgütertransfer (KGTG) vom 23. Juni 2003 (BB1 2003 4475). 
Beide Räte folgten dem Antrag des Bundesrates, die UNESCO-Konvention von $1970 \mathrm{zu}$ ratifizieren. Die Ratifizierungsurkunden wurden von Bundesrätin Micheline Calmy-Rey anlässlich der UNESCO-Generalkonferenz ${ }^{25}$ am 1 . Oktober 2003 in Paris hinterlegt. Bei dieser Gelegenheit betonte die Bundesrätin, die Schweiz leiste mit der Ratifizierung einen wesentlichen Beitrag an die Bewahrung des Kulturerbes der Menschheit und bestätige ihre Entschlossenheit, einen fairen und offenen Kulturaustausch zu führen.

\section{$\square$ Plünderung des kulturellen Erbes im Irak - Position der Schweiz.}

Die Plünderungen des archäologischen Museums von Bagdad und der irakischen Nationalbibliothek Mitte April 2003 lösten in der internationalen Öffentlichkeit grosse Besorgnis aus. Möglicherweise haben diese Ereignisse die Beratungen des Parlaments über die Annahme des KGTG mit beeinflusst. Sobald die Meldungen über die Plünderungen publik wurden, ergriff die internationale Gemeinschaft unter der Schirmherrschaft der UNESCO Massnahmen, um den Handel mit Kulturgütern aus dem Irak zu unterbinden. Mitte April warnte das Bundesamt für Kultur die betroffenen Kreise vor der Gefahr, dass irakische Kulturgüter in der Schweiz auftauchen könnten, und empfahl nachdrücklich, Kulturgüter mit nicht nachgewiesener Herkunft weder zu kaufen, noch entgegenzunehmen ${ }^{26}$. Ende Mai 2003 verbot der Bundesrat in einer Verordnung den Handel mit irakischen Kulturgütern und ergriff Massnahmen zur Erleichterung der Rückführung irakischer Kulturgüter. Laut der Verordnung werden die Ein-, Durch- und Ausfuhr sowie der Verkauf, der Vertrieb, die Vermittlung, der Erwerb und die anderweitige Übertragung von irakischen Kulturgütern, die seit dem 2. August 1990 in der Republik Irak gestohlen wurden, gegen den Willen des Eigentümers abhanden gekommen sind oder rechtswidrig aus der Republik Irak ausgeführt wurden, verboten. Diese Massnahme gilt auch für Kulturgüter aus Raubgrabungen. Die rechtswidrige Ausfuhr eines Kulturguts wird vermutet, wenn dieses sich nach dem 2. August 1990 nachweislich in der Republik Irak befunden hat ${ }^{27}$.

Als konkretes Zeichen des Engagements für die irakischen Kulturgüter steuerte das Eidgenössische Departement für auswärtige Angelegenheiten 250'000 Dollar zur von Italien und Japan mit unterstützten UNESCO-Initiative bei, welche die Schaffung einer Datenbank über die irakischen Kulturgüter bezweckt.

Ferner ist zu erwähnen, dass der Bundesrat im Sommer 2003 die Botschaft ${ }^{28}$ über das Zweite Protokoll vom 26. März 1999 zum Haager Abkommen ${ }^{29}$ von 1954 für den Schutz von Kulturgut bei bewaffneten Konflikten genehmigte. Das

25 Bundesamt für Kultur, Ratifikation der UNESCO-Konvention von 1970 durch die Schweiz, Pressemitteilung, 1. Oktober 2003.

26 Bundesamt für Kultur, Plünderung des kulturellen Erbes im Irak, Pressemitteilung, 16. April 2003.

27 Verordnung vom 28. Mai 2003 über Wirtschaftsmassnahmen gegenüber der Republik Irak (AS 2003 1887), mit welcher die gleichnamige Verordnung vom 7. August 1990 (SR 946.206) geändert wird.

28 Botschaft über das Zweite Protokoll vom 26. März 1999 zum Haager Abkommen von 1954 für den Schutz von Kulturgut bei bewaffneten Konflikten, 20. August 2003 (BB1 2003 6091).

29 Beim Abkommen für den Schutz von Kulturgut bei bewaffneten Konflikten, das am 14. Mai 1954 in Den Haag angenommen wurde, handelt es sich um ein Vertragswerk zur Verhütung von unersetzlichen Schäden und Verlusten, wie sie den Kulturgütern während des Zweiten Weltkriegs zugefügt wurden. Das unter der Ägide der UNESCO entstandene Haager Abkommen ist als erstes weltweit anerkanntes Instrument ausschliesslich dem Schutz der Kulturgüter gewidmet. 
im Rahmen der UNESCO ausgearbeitete Zweite Protokoll greift die Entwicklungen im humanitären Völkerrecht, im internationalen Strafrecht und im Recht betreffend den Schutz des kulturellen Erbes auf. Die Schweiz spielte als Vertragspartei des Haager Abkommens und des Ersten Protokolls eine Schlüsselrolle bei der Ausarbeitung des Zweiten Protokolls. Die Ratifikation des Protokolls erforderte keine Anpassung der schweizerischen Gesetzgebung.

Jahrbuch 1999, ,Regelung des Kunsthandels in der Schweiz“, S. 201-202; 1997, Catherine Schümperli Younossian, „Handel und Verkehr mit Kulturgütern: Stand der Regelung in der Schweiz“, Analysen und Stellungnahmen, S. 277-288.

\section{QUELLEN}

Bundesrat, Botschaft über das Zweite Protokoll vom 26. März 1999 zum Haager Abkommen von 1954 für den Schutz von Kulturgut bei bewaffneten Konflikten, 20. August 2003 (BB1 2003 6091).

Bundesrat, Botschaft über die UNESCO-Konvention von 1970 und das Bundesgesetz über den internationalen Kulturgütertransfer (KGTG) vom 21. November 2001 (BB1 2002 535).

Nationalrat und Ständerat, Amtliches Bulletin, Session 2003, <www.parlament.ch/ab/frameset/index. htm $>$.

Eidgenössisches Departement des Innern, Kulturgütertransfergesetz. Bericht über die Ergebnisse des Vernehmlassungsverfahrens, Bern, Bundesamt für Kultur, November 2001.

Eidgenössisches Departement des Innern, Bericht der Arbeitsgruppe Internationaler Kulturgütertransfer liegt vor, Pressemitteilung, 26. August 1998.

Oberzolldirektion, Schweizerische Aussenhandelsstatistik. Jahresbericht 2002, Bern, 2003.

Bundesgesetz, über den internationalen Kulturgütertransfer (KGTG) vom 20. Juni 2003, BB1 2003, 4475).

Bundesamt für Kultur, „Regeln für ein Milliardengeschäft ?“, BAK-Journal, 5/2002.

Bundesamt für Kultur, Plünderung des kulturellen Erbes im Irak, Pressemitteilung, 16. April 2003; Ratifikation der UNESCO-Konvention von 1970 durch die Schweiz, Pressemitteilung, 1. Oktober 2003.

Bundesamt für Kultur, Internationaler Kulturgütertransfer. UNESCO-Konvention von 1970 und UnidroitKonvention von 1995, Bericht der Arbeitsgruppe, Nr. 304.250 d, Bern, 1998.

Parlament, Zusammenfassung 01.077. Kulturgütertransfergesetz, 30. Juli 2003.

Guex S. und Lafontant Vallotton C. (Hrsg.), Le marché suisse de l'art (XIX et XX siècles), Traverse. Revue d'histoire, Nr. 1, 2002.

Moulin R., Le marché de l'art, coll. Champs, Paris, Flammarion, 2003.

Le Temps, „Internet et Interpol volent au secours du patrimoine culturel africain“, 31. Juli 2000 ; „Marché de l'art: une loi qui criminalise ou responsabilise les marchands“, 28. Januar 2003; „Biens culturels et biens publics“, dossier du Samedi culturel, 1. März 2003; „Visite guidée dans les ports francs de Genève“, 24. November 2003.

Neue Zürcher Zeitung, D. Cahn und A. Gaiser, „Unidroit, Diebstahl und illegaler Export“, 23. Januar 1999.

\section{INTERNET-ADRESSEN}

Schweizerische UNESCO-Kommission: <www.unesco.ch>.

Erklärung von Bern: <www.EvB.ch>.

Internationales Institut zur Vereinheitlichung des Privatrechts (Unidroit) : <www.unidroit.org $>$.

Bundesamt für Kultur >Sektion Kulturgütertransfer : <www.kultur-schweiz.admin.ch $>$.

Organisation der Vereinten Nationen für Erziehung, Wissenschaft und Kultur (UNESCO): <www. unesco.org $>$.

Parlament: <www.parlament.ch>. 